\title{
3D Reconstruction Method Based on Binary coded Pattern
}

\author{
JaeWook $\mathrm{Ha}^{1}$, Soon Kwon ${ }^{1}$, Jaekyo Jeong ${ }^{1}$ and HyunWoo Kim ${ }^{1}$ \\ Dept. Of IT-Convergence, Daegu Gyeongbuk Institude of Science \& Technology \\ (DGIST) \\ \{jwha, soonyk, jekyo,kimhw2109\}@dgist.ac.kr
}

\begin{abstract}
Three-dimensional (3D) reconstruction based on a coded structured light method is a widely used technique to recover objects surfaces. This technique is based on projecting a pattern onto object to reconstruct and the illuminated scene is imaged by calibrated camera. The pattern creates an artificial features on the surfaces of objects and can be a correspondence points. The $3 D$ information is reconstructed by triangulating the correspondence between projected pattern and decoded pattern in imaged scene. In this paper, 3D reconstruction method based on a coded structured light is proposed. The pattern is encoded in binary pattern and it has uniqueness in the search range to find correspondence points. The pattern is projected using an infrared laser and a diffraction optical element (DOE) for the invisible areas. Then, the illuminated scene is imaged by a sensitive camera. As a result, the disparity of correspondences between matched points draw a $3 D$ surface information.
\end{abstract}

Keywords: $3 D$ reconstruction, coded structured light, binary coded pattern

\section{Introduction}

Three-dimensional (3D) reconstruction is an important topic in computer vision because it is used in numerous applications such as remote sensing, object recognition, industrial inspection, and robot systems, among others. Generally, 3D reconstruction methods are categorized into passive and active techniques. In passive techniques, the scene is imaged using camera from two or more points of view and then the correspondence between the images is found. Then, the correspondence is triangulated and the 3D position is obtained [1]. However, it is difficult to find correspondence between images from different cameras, even when considering the epipolar constraints [5]. Therefore, the passive techniques are limited in textureless scenes due to the problem of finding correspondence [3,4]. In contrast, active techniques replace one of the two cameras with a device that projects a pattern onto the object surface and the scene is captured using one or more cameras. The projected patterns create an artificial feature on the object's surface that can be used as correspondence, particularly in textureless scenes [6]. Then, 3D reconstruction is possible when looking for differences between the projected patterns and imaged patterns. This is similar to passive techniques [15].

The structured light scheme differs according to the type of pattern and projection method such as time division pattern projection, coded color patterns, and coded patterns [9]. The time division pattern is reliable and accurate for dense 3D reconstruction, but it cannot be used for real time or moving target $3 \mathrm{D}$ reconstruction 
[10]. In coded color patterns, the 3D reconstruction results are affected by the object and background colors [11]. Coded patterns can reconstruct 3D surfaces for moving targets and independent colors, but it requires a decoding step for pattern recognition to match the projected pattern [12].

In this paper, a coded structured light method is proposed for 3D reconstruction. The pattern is coded for binary representation and projected using an infrared laser and a diffraction optical element (DOE) for the invisible areas [18]. The invisible pattern in the scene is imaged using a sensitive camera. Then, after pattern recognition, the patterns from the projected pattern and the recognized pattern are matched in order to reconstruct the $3 \mathrm{D}$ surface.

The following section introduces the reconstruction method. In 2.1 Section, the infrared environment is introduced. And in the next section, the image preprocessing is described to pattern detection. Then the white pattern recognition and matching method for 3D reconstruction is presented in Section 2.2. Then, the results with the disparity of correspondence are presented in Section 3, and the paper is concluded in Section 4.

\section{Reconstruction Method}

In Structured light method, the Pattern encoding method is very important. Because the type of pattern affects the structured light performance including the resolution, speed, and accuracy [9]. Time multiplexing has an advantage in dense 3D reconstruction; however, it cannot reconstruct moving targets [7, 8]. Coded color patterns have a disadvantage if the target has a similar color to the colored pattern [13]. The pattern consists of a specific shape, then it may also be affected by the objects and background shapes. If shape of objects is similar to the projected pattern, the pattern recognition in imaged scene is difficult. However, coded patterns can reconstruct a moving target in 3D; even if it is represented using binary symbols, it is independent of the color, shape, and other details from the background and objects [2, 17].

However Coded patterns have a disadvantage in pattern decoding. Therefore, pattern coding methods, such as the pseudorandom, M-array, perfect map, and De Bruijn methods, have been investigated for a long time in order to facilitate the pattern decoding $[14,16]$. These pattern encoding methods create symbols for uniqueness within a specific range. The projected pattern is compared with the imaged pattern for recognition [19]. If the pattern is repeated within the search range, matching errors occur. Despite these methods, the light source remains visible and it is difficult to subtract the pattern from the image.

\subsection{Advantage of Infrared Environment}

This research used a coded binary pattern that was projected using an infrared laser and DOE. Then the illuminated scene is imaged by a sensitive camera. As a result, in the imaged scene, there was a small object shape and the pattern is clearly represented. It reduces interference from shape of object when pattern in imaged scene is recognized. And it is effective to extract the pattern from imaged scene and pattern recognition. The first step of $3 \mathrm{D}$ reconstruction is a pattern detection from the imaged scene to match a projected pattern. In infrared environment, the pattern is represented clearly and it is extracted

Figure 1 presents the image captured using the sensitive camera in infrared light. Clear patterns and shadows can be seen in the image. The background effect is 
eliminated and the object is recognized using the shape of the shadow. There is also a material effect: because the material of the cylindrical object has a lower level of light reflection, the projected pattern is faint and, in some areas, invisible. In this case, the pattern does not appear despite the visible light.

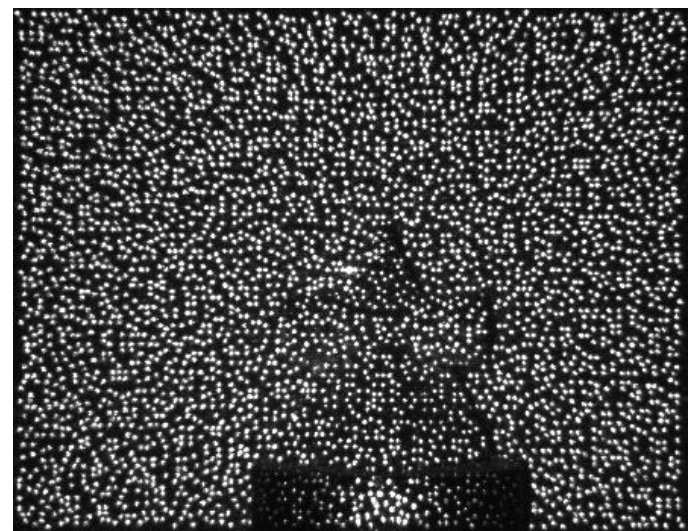

\section{Figure 1. Image captured using a sensitive camera with a coded pattern that was projected using an infrared laser and DOE}

Following chapter introduce a proposed $3 \mathrm{D}$ reconstruction method in this paper. The first step of $3 \mathrm{D}$ reconstruction is a pattern detection from the imaged scene to match a projected pattern. Then, the pattern in imaged scene is recognized by matching between detected pattern and projected pattern. Lastly, disparity map is calculated from difference of matching points.

\subsection{Pattern Matching Method}

In order to reconstruct the $3 \mathrm{D}$ image, the surface is triangulated using the difference between the projected pattern and the imaged pattern. The difference is represented using the shift of correspondence points on the epipolar constraints. In this research, the correspondence points are dots that have symbols. The symbols consist of white dots and black dots. The white dots are recognized easily, but black dots are difficult to distinguish from the background. Therefore, the black dots recognition uses the results of the white symbols.

In order to match the white dots to projected pattern, a sliding window method was used. The projected symbols are unique in the specific search range. And the pattern is designed to maintain the uniqueness of the symbols with a specific size in the search range. The size of the sliding window is the same as that of the symbol. The five steps for matching the symbols are as follows.

1. A symbol is extracted from the pattern in the image to the window.

2. The symbol in the projected pattern is extracted to the window.

3. The matching cost between the windows is calculated.

4. The window slides along the search range in the projected pattern and Steps 2 and 3 are repeated until the symbol that has the maximum matching cost is found. 
5. A white dot in the center of the window is matched to the one in the window that has the maximum matching cost.

The matching cost between the windows is calculated when the proportion of the correct position of the white and black dots is more than an arbitrary threshold value. If the proportion is less than the threshold value, the symbol is regarded as a different symbol to the one being sought. In contrast, the matching cost is the proportion of dividing the number of correction positions of white dots by the number of white dots in the window.

The first step is an extraction of white dots. To extract the white dot symbols from imaged scene, an image preprocessing methods are performed. And then detected white dot pattern is recognized. Following Figure 2 and 3 show input image and result of image preprocessing.

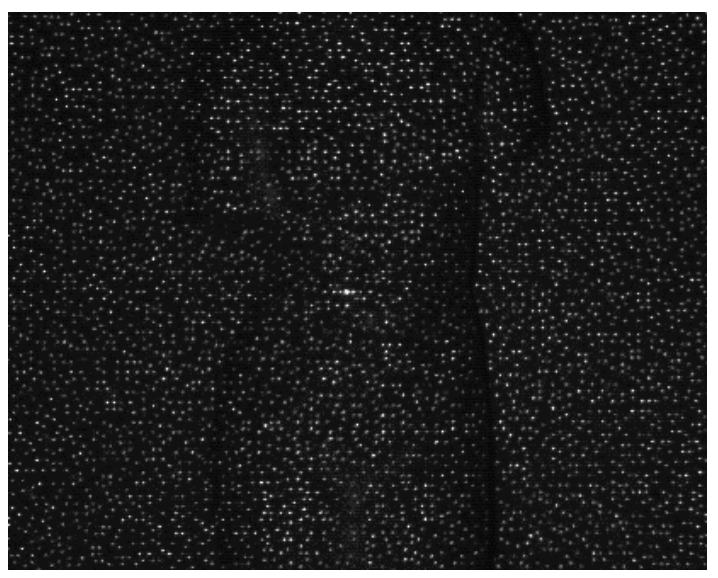

\section{Figure 2. The input image to reconstruct 3D information}

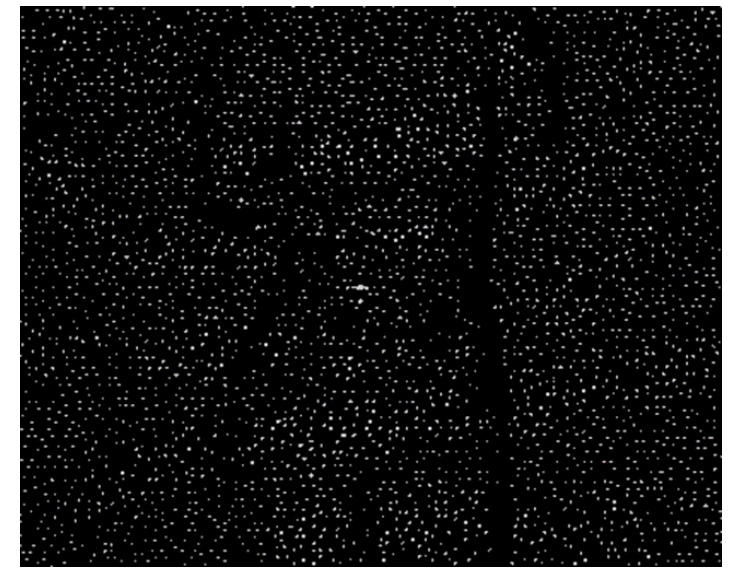

Figure 3. The image of extracted white pattern

From extracted white dot symbols, pattern matching is performed between projectede and detected one. However, the matching between projected pattern and extracted pattern is difficult. Because the projector and camera is not calibrated and they have a 
different domains. So white dot symbol recognition is needed. Following Figure 4 shows the result of recognition from white dot symbols.

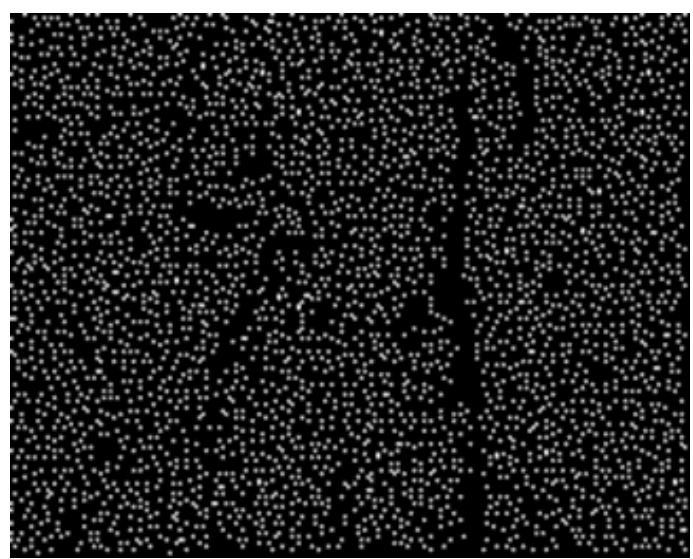

\section{Figure 4. The white pattern recognition result}

\subsection{Disparity Map Reconstruction}

After the white dots is recognition, a sliding window method is performed to match between project pattern and recognized white symbols. There are two sliding window. First one slides in projected pattern and extract symbols. Second one slides in recognition symbols and extracts. When the difference between windows is minimum, the matching cost can be maximum. Then the recognized symbol is matched.

Every case of windows are sliding through pattern and symbols, matching cost is calculated. Following Figure 5 show the result of matching between reference and recognized symbols.

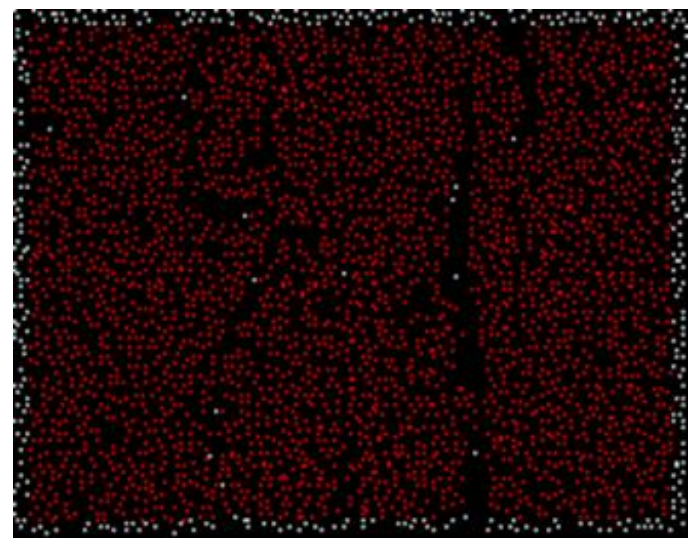

Figure 5. The matching result between projected pattern and recognized symbols

After the white dots are matched, the black dots are matched using the positions of the matched white dots. The black dots are matched along the projected pattern. To a black symbol matching, a symbol is first extracted from the projected pattern to the window where the black dot is centered. Then, a symbol is extracted from recognized 
pattern to another window. The window for extracting symbol in recognized pattern is sliding through a search range. A black dot is also centered in another window. To matching between windows, a matching cost is calculated. The matching cost has a weighting value in white dot position because the white dot position is a result of a matching points whereas black position is not correct.

Finally, disparity is calculated to make disparity map. All of matched points between projected pattern and recognized pattern from imaged scene are subtracted. And then, the difference reconstructs a 3D surface.

\section{Experimental Results}

The following results demonstrate a disparity image using the sliding windows matching method with a coded pattern. The experimental environments were as follows.

1. The positions of the projector and camera were collinear.

2. The projector consists of an infrared laser and diffraction optical elements.

3. The devices placed at an indoor environment because the sun light has all of frequency band.

4. The object position was $1.5 \mathrm{~m}$ from the camera and projector.

The collinear position of camera and projector is very important. Generally, the difference position in the line of sight (LOS) takes horizontal movements in image captured by camera. However, if the position of camera and projector is not collinear, horizontal and vertical movements are occurred. Then, disparities between projected pattern and imaged pattern are diagonal. And if the vertical movements is not an equal especially vertical direction through imaged pattern, disparities calculation is hard. The Sun light has all bands of frequency and it overwhelm the infrared pattern. In outdoor environment, the pattern in imaged scene is disappeared.

Following Figure 6 and 7 the disparity images that illustrate the difference between the symbols in the projected pattern and the symbols recognized in the imaged pattern. The 3D reconstruction is drawn through applying real distance to the disparity. Despite the holes and shadows due to mismatching and false detection, the quality of disparity image is acceptable. The shadowed areas are based on the projector illumination and the quality of material. In left image of Figure 6, the symbol on the object under the geometrical figure is not recognized; furthermore, symbols are not present in the left side of the bust sculpture in left image of Figure 7. The quantity of dots in the disparity image is described in Table 1.

Table 1. Number of matched dots for projected dots and imaged dots

\begin{tabular}{llllll}
\hline Scene & Projected dots & $\begin{array}{l}\text { Projected } \\
\text { white dots }\end{array}$ & Matched dots & $\begin{array}{l}\text { Recognized } \\
\text { white dots }\end{array}$ & $\begin{array}{l}\text { Matched white } \\
\text { dots }\end{array}$ \\
\hline $\begin{array}{l}\text { Geometrical } \\
\text { figure }\end{array}$ & 34,815 & 3860 & 28,116 & 3204 & 2907 \\
$\begin{array}{l}\text { Bust sculpture } \\
\text { 34,815 }\end{array}$ & 3860 & 24,586 & 3086 & 2688 \\
\hline
\end{tabular}

Table 1 presents the detection, recognized and matching results of the total number of projected dots and matched dots. In infrared environment, the background effects is reduced. There are a pattern and shadow of object shape. In spite of an exception from 
outside symbols in image, the recognition result shows a high performance. The recognition result lead in high performance of a matched white symbol. As a result, black symbols decoding is performed through positions of white symbols matching result. The matching proportion is approximately $80.75 \%$ and $70.61 \%$ despite the holes and shadow effects. For the detected white dots, the performance increased to approximately $90 \%$ and $87 \%$. As a result, most recognized symbols were matched with the projected symbols.

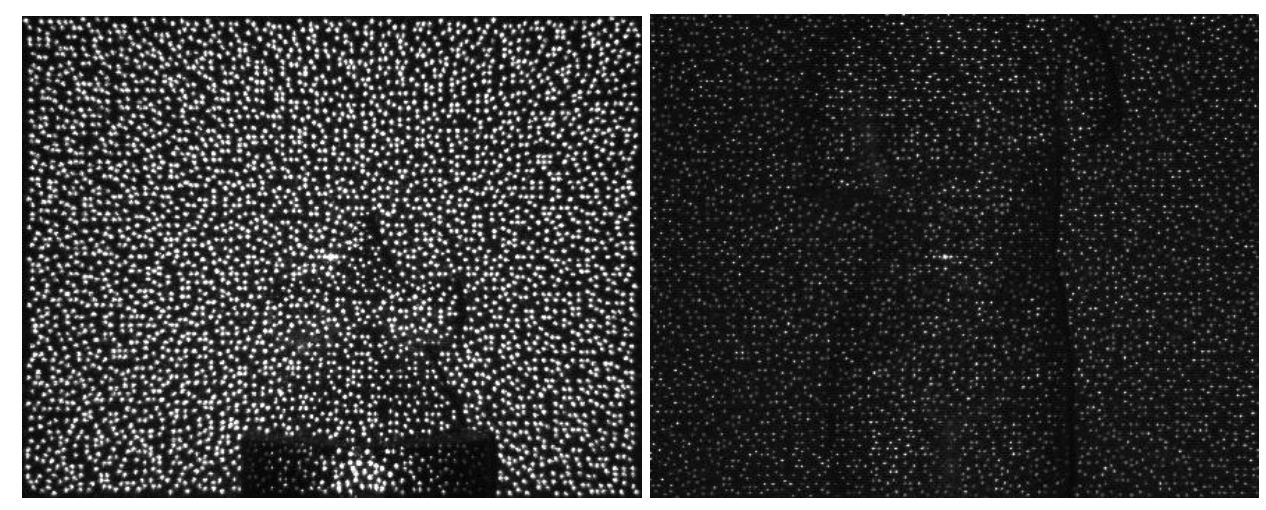

Figure 6. An input images captured using a sensitive camera for the geometrical figure
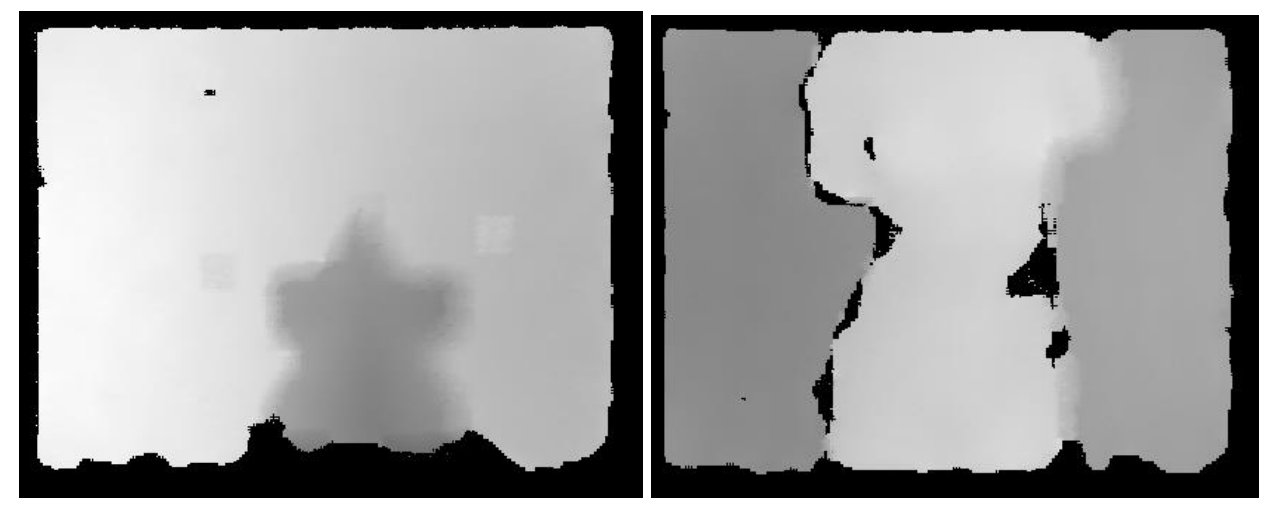

\section{Figure 7. The Result of the disparity between the matched projected patterns in figure 6}

\section{Conclusion}

The proposed coded structured light method projects a pattern onto an object in order to reconstruct its 3D surface. Unlike passive methods, the coded structured light creates an artificial texture that can be used to reconstruct the 3D surface. The coded structured light can also reconstruct a 3D surface for moving targets. In infrared conditions, the color of the object and background do not affect the pattern recognition.

This paper proposed a method for $3 \mathrm{D}$ reconstruction using coded structured light in infrared conditions. A pattern is designed through binary encoding method because the coded pattern can be real-time processing. And the pattern consists of binary dots to eliminate background color effects. Even if the pattern is projected by an infrared laser 
and DOE. The infrared has advantages in invisible in human and elimination of object shape effect to pattern recognition. A scene is captured using a sensitive camera, and the pattern in the image is recognized.

In order to determine the difference between the projected pattern and the imaged pattern, a sliding window method that is the same size as the pattern symbol was used. First, the white symbol is extracted from imaged pattern and projected pattern, then they are compared by matching cost. Secondly, the black symbols were matched using the matching information from the white dots. The black symbol is also extracted to sliding window and the position of white dots in window is used to match between black symbol in projected pattern and black symbol in recognized pattern. Finally, the difference was calculated using the position of the matched dots. It can be disparity to reconstruct a $3 \mathrm{D}$ surface.

This paper illustrated the disparity images from the results of the matching performance. Despite the holes and shadows where the white dots were not visible, the total matching performance was more than $70 \%$ and the white dot matching performance was more that $87 \%$ for verification in the visible areas. This paper demonstrates the possibility of reconstructing the 3D surface using coded structured light in infrared conditions.

\section{Acknowledgements}

This work was supported by the DGIST R\&D Program of the Ministry of Education, Science and Technology of Korea (grant no. 13-NB-05).

\section{References}

[1] J. Salvi, X. Armangué and J. Batlle, "A comparative review of camera calibration methods with accuracy evaluation", Pattern Recognition, vol. 35, (2002).

[2] J. Salvi, J. Pagés and J. Batlle, "Pattern codification strategies in structured light systems", Pattern Recognition, vol. 37, (2004).

[3] Z. Zhang, "Flexible Camera Calibration By Viewing a Plane From Unknown Orientations", Computer Vision, The Proceedings of the $7^{\text {th }}$ IEEE International Conference, ICCV, (1999).

[4] H. Kawasaki, R. Furukawa, R. Sagawa and Y. Yagi, "Dynamic scene shape reconstruction using a single structured light pattern", IEEE Conference on Computer Vision and Pattern Recognition, CVPR, (2008).

[5] J. Batlle, E. Mouaddib and J. Salvi, "Recent Progress in coded structured light as a technique to solve the correspondence problem: a survey", Pattern Recognition, vol. 35, (1998).

[6] J. Salvi, J. Batlle and E. Mouaddib, "A robust-coded pattern projection for dynamic 3D scene measurement", Pattern Recognition Letters, (1998).

[7] D. Scharstein, R, Szeliski, "High-Accuracy Stereo Depth Maps Using Structured Light", IEEE Computer Society Conference on Computer Vision and Pattern Recognition, CVPR, (2003).

[8] R. Sagawa, Y. Ota, Y. Yagi, R. Furukawa and N. Asada, "Dense 3D reconstruction method using a single pattern for fast moving object", IEEE $12^{\text {th }}$ International Conference on Computer Vision, ICCV, (2009).

[9] J. Geng, "Structured-light 3D surface imaging: a tutorial", IEEE Intelligent Transportation System Society, Advances in Optics and Photonics, vol. 3, (2011).

[10] I. Ishii, K. Yamamoto, K. Doi and T. Tsuji, "High-speed 3D Image Acquisition Using Coded Structured Light Projection”, IEEE/RSJ International Conference on Intelligent Robots and Systems, (2007) October 29 - November 2, San Diego, USA.

[11] P. Payeur and D. Desjardins, "Structured Light Stereoscopic Imaging with Dynamic Pseudo-random Patterns", Image Analysis and Recognition Lecture Notes in Computer Science, (2009).

[12] P. Lavoie, D. Ionescu and E. J. Petriu, "3-D Ojbect Model Recovery From 2-D Images Using Structured Light", IEEE Transaction on Instrumentation and Measurement, (2004).

[13] P. M. Griffin, L. S. Narasimhan and S. R. Yee, "GENERATION OF UNIQUELY ENCODED LIGHT PATTERN FOR RANGE DATA ACQUISITION”, Pattern Recognition, (1992). 
[14] T, Etzion, "Construction for Perfect Maps and Pseudorandom Arrays", IEEE Transaction on Information Theory, (1988) September.

[15] F. Zhou and G. Zhang, "Complete calibration of a structured light stripe vision sensor through planar target of unknown orientations", Image and Vision Computing, vol. 23, no. 1, (2005).

[16] G. Isaak, "Constructions for higher dimensional perfect multifactors, aequationes mathematicae", vol. 8, no. 2, (2002) August.

[17] K. J. Priya and R. S. Rajesh, "A Local Min-Max Binary Pattern Based Face Recognition using Single Sample per class".

[18] D. Kim, S. Lee and J. Paik, "Active Shape Model-Based Gait Recognition Using Infrared Images", International Journal of Signal Processing, Image Processing and Pattern Recognition, (2009).

[19] J. Zhou, T. Xu and J. Gan, "Facial Expression Recognition Based on Local Directional Pattern Using SVM Decision-level Fusion", International Journal of Database Theory and Application, (2013). 
International Journal of Signal Processing, Image Processing and Pattern Recognition Vol.6, No.6 (2013) 